\title{
El delito de robo con fuerza en vivienda en Cataluña: Un estudio criminológico
}

Manuscrito recibido el 26 de abril de 2010 / Publicado el 07 de junio de 2010

Francesc Reales

Subinspector de la Policía de la Generalitat-Mossos d'esquadra

\section{RESUMEN}

Dentro de los delitos contra el patrimonio y el orden socioeconómico se encuentra el robo en domicilio, este agravante del delito de robo con fuerza, de gran repercusión mediática, aumenta y disminuye a lo largo de diversos periodos temporales y debido a multiplicidad de causas. En el estudio empírico que aquí se presenta se analizan diversas variables tales como, la inmigración, el paro, la construcción de viviendas..etc. en unas determinadas áreas geográficas de Cataluña y durante el periodo 2000-2008. A la conclusión que se llega es que, determinadas variables sí favorecen la comisión de este delito, mientras que otras aportan poco al desarrollo del hecho delictivo.

Palabras clave: Robo en vivienda; Delincuencia; Evolución criminalidad, Victimización, Criminología.

\begin{abstract}
Burglary is one of the main crimes committed against property and the socioeconomic order in Spain. It is aggravated and attracts greater attention from the mass when it is accompanied by the use of force or violence. For a number of reasons, the incidence of burglary tends to increase and decrease over time. In this empirical research, several variables, including immigration, unemployment and housing construction are presented and analyzed. Burglary is studied in specific areas of Catalonia and in the period 2000-2008. The main conclusion reached is that some variables have a considerable influence on the likelihood of this crime being committed, whereas others make relatively little contribution to this type of crime.
\end{abstract}

Key words: Burglary, Criminality, Evolution of Crime, Victimization, Criminology. 


\section{Introducción.}

Sin duda, uno de los delitos que más sensación de inseguridad generan en la sociedad es el robo en vivienda, no solo por el valor de lo sustraído sino, y en mayor medida, por la agresión a la intimidad que supone la intrusión de un extraño en un ámbito tan personal como es el hogar.

El objetivo de este artículo es analizar la evolución del delito de robo en vivienda en cuatro comarcas catalanas: el Segrià, el Baix Empordà, el Gironès y la Cerdaña. Se observa la evolución de los delitos contra el patrimonio relacionados con el robo en vivienda para estudiar si la tendencia es la misma que en los últimos años, es decir, una tendencia descendente tanto en Cataluña, en España y en el resto de países europeos, excepto contadas excepciones ${ }^{1}$. Se observa igualmente si, por el contrario, esta tendencia a la baja se rompe a finales de la década para iniciar un aumento en el número de delitos conocidos. Así pues, se presenta una visión actual del delito en dichas comarcas y su evolución en el periodo 2000-2008. Se analiza, mediante los datos recogidos, si en los delitos contra el patrimonio y en el robo en el interior de vivienda en concreto, en los últimos años ha cambiado substancialmente la tipología del autor vinculada a la creciente inmigración acontecida en nuestro país. Se estudia si esta inmigración ha modulado, de manera destacable, las características de estos tipos de robo con el cambio en la ejecución y si ha propiciado un incremento de la sensación de inseguridad, hecho que ha generado un incremento de la alarma social como respuesta a estos tipos delictivos ${ }^{2}$. También se estudia la evolución de la población inmigrada, la evolución del número de viviendas construidas, el número de detenidos, su nacionalidad y finalmente, el número de efectivos policiales en cada año estudiado.

\footnotetext{
${ }^{1}$ Segun "The Burden of Crime in the EU, A Comparative Analysis of the European Survey of Crime and Safety (EU ICS) 2005". p.23 Bélgica, es el único país de los 18 europeos que analiza este informe, que no ha mostrado una disminución de sus índices de delincuencia.

2 Un análisis sobre inseguridad y delito lo encontramos entre otros en (Hale 1996), (Medina 2003) y (Fernández-Ramírez 2008). Fernández-Ramírez plantea una interesante revisión sobre el tema a la vez que presenta una reflexión teórica para superar la "aparente sensación de agotamiento conceptual de la investigación sobre delincuencia e inseguridad".
} 
La especial protección otorgada al lugar dónde viven personas se ha manifestado a lo largo de toda la historia jurídica. Se podría considerar como causas de la agravación del delito en casa habitada un conjunto de fundamentaciones complementarias entre sí. En primer lugar, encontramos un peligro para la víctima puesto que, el riesgo de transformación del delito en un robo violento se encuentra en la habitabilidad del lugar. La presencia de sus ocupantes o su resistencia puede dar lugar a actos lesivos para la vida o la integridad física. La jurisprudencia suele defender una doble fundamentación, por una parte, el riesgo adicional para otros bienes jurídicos; como la vida o integridad física...etc. y por otra parte, y en segundo lugar, la profanación propia del hogar que comporta un impacto psicológico en la víctima.

En tercer lugar podemos hablar de una peligrosidad criminal, ya que, desde el punto de vista criminológico, podríamos decir que en el robo con fuerza en las cosas y en el robo violento actúa "una voluntad criminal más fuerte e intensa que en un hurto". Esto se debe a que, a menudo, se deben superar considerables obstáculos, ya sea de tipo material, para el caso del robo con fuerza, como la de anular la resistencia de una persona, en el caso del robo con violencia o intimidación (Middendorf 1973).

En gran cantidad de hechos delictivos se requiere habilidad mecánica, fuerza corporal y técnica. El hecho de utilizar técnicas específicas de superación de los obstáculos y la utilización de utensilios necesarios para su fin, ponen de manifiesto que estas modalidades de sustracción exigen para su ejecución una preparación detallada.

Desde el punto de vista de la victimología es destacable la aportación que hacen los ciudadanos a este tipo de delito. (Landrove-Diaz 1990) indica que hay delitos en los cuales la víctima no aporta nada a su desarrollo (víctima fungible), y hay otros en los cuales la víctima participa con un papel más o menos activo (víctimas infungibles o participantes). ${ }^{3}$ Así pues, la actitud que tome la persona en el momento de proteger adecuadamente o poniendo difícil el acceso a su domicilio, propiciará en gran medida que sea finalmente víctima o no de este hecho delictivo.

\footnotetext{
${ }^{3}$ como por ejemplo en la omisión de las precauciones más elementales y así favoreciendo la comisión del hecho delictivo (no cerrando la puerta de la vivienda o dejando las llaves bajo la alfombra ante la puerta).
} 


\section{Estado de la cuestión}

La investigación criminológica de carácter cuantitativo en el tema del robo en vivienda no ha sido muy desarrollada ni en Cataluña ni en el Estado español, salvo las aportaciones hechas por el Instituto andaluz interuniversitario de Criminología. Este instituto con la publicación de su Boletín Criminológico ha dado paso a estudios de sobrado interés en el ámbito del estudio del robo en vivienda como por ejemplo: "La delincuencia en todas las capitales andaluzas" de Elisa García España, Fátima Pérez Jiménez y Maria José Benítez Jiménez o el estudio de "La delincuencia en Córdoba, Huelva y Sevilla según las víctimas" de las mismas autoras. Estos estudios, que se apoyan en encuestas a ciudadanos para conocer los índices reales de delincuencia, han abordado el problema del robo en vivienda como un apartado dentro de un contexto más general de los delitos patrimoniales y no como un estudio concreto de este delito. Como ejemplo, el Observatorio de la delincuencia en Andalucía, a partir de los datos analizados recogidos el año 2005, nos daban una tasa de victimización de un 0,8 en robos en vivienda en la ciudad de Málaga, situándose muy por debajo del 2,3 del año 1994. Este Observatorio de la delincuencia en Andalucía, creado en el seno del Instituto andaluz ínter universitario de Criminología (sección de Málaga), financiado por la Fundación El Monte, es un centro de investigación y documentación especializado en el estudio de la evolución de la delincuencia en la Comunidad Andaluza y en España. Hasta la fecha ha publicado tres informes sobre la delincuencia en el estado español: "Evolución de la delincuencia en España y Andalucía: Análisis e interpretación de las estadísticas oficiales" (Informe ODA, 2004), "Seguridad ciudadana y actuaciones policiales". (Informe ODA, 2005), y "La delincuencia según las víctimas" (Informe ODA, 2006) (los tres informes elaborados por (García España et al. 2006).

Este observatorio quiere ser una fuente de información a partir de datos oficiales e instituciones sobre la delincuencia y realizar estudios empíricos sobre varios aspectos del fenómeno delictivo. Los informes del Observatorio de la delincuencia en Andalucía analizan desde 2003 la situación penal y penitenciaria nacional y autonómica andaluza desde varios aspectos. Uno de los estudios más próximos a la investigación que se aborda es el que trata la "Reciente evolución de la delincuencia en Andalucía"(García España and Pérez Jiménez 2004), que muestra, como su título indica, la evolución de 
diferentes delitos en la comunidad autónoma andaluza. Para ver su evolución, las autoras analizan los datos de los anuarios del Ministerio del Interior, el informe del Sindicato Unificado de Policía en Andalucía y los resultados del Centro de Investigaciones Sociológicas en materia de seguridad ciudadana. En este estudio se analizan los datos de 1998 a 2003 y en materia de delitos contra el patrimonio, las autoras muestran un descenso desde el año 2001 en esa comunidad autónoma.

Según el Ministerio del Interior () en el año 2008 hay varios delitos que aumentan, entre ellos, los robos con fuerza en vivienda que en 2008 llegaron a niveles de 2003, tras varios años de descenso. Según Interior, el robo en vivienda en España ha aumentado; la evolución de los datos de criminalidad en el pasado año han lanzado un dato nuevo: los robos con fuerza en viviendas han crecido un cuatro por ciento y se sitúan en un 37,1 por ciento, sobre una tasa de 10.000 viviendas, respecto al 33,2 de 2007. El incremento del bienestar(Avilés Ferré 2003) se traduce en un aumento de las oportunidades para la comisión de delitos, especialmente contra la propiedad. La proliferación de vehículos particulares, de segundas residencias, los viajes en los cuales queda vacía la vivienda habitual ofrecen otras tantas oportunidades de delito. Así pues, el autor indica que nos encontramos en un incremento de la demanda de seguridad debido al desarrollo, con la diferencia que, en este caso, se produce también un incremento del delito real.

(Ruiz 2006) elabora un estudio de los delitos contra el patrimonio en referencia a la ciudad de Badajoz dónde también se fija en los delitos contra la vivienda. En su investigación hace un análisis de los autores, y de su forma de actuar, en la comisión del delito mediante datos policiales y sentencias dictadas durante tres años por el juzgado penal de Mérida. El autor, con datos de finales de los 90 y comienzos del 2000, muestra el aumento que ha experimentado el robo en vivienda en la provincia estudiada. Este aumento de hechos delictivos contra la vivienda y contra el patrimonio en general lo presenta también (García España and Pérez Jiménez 2004)en su estudio sobre España y Andalucía.

A nivel internacional el panorama cambia significativamente, puesto que, en los países anglosajones, (preferentemente los EE.UU. y el Reino Unido) se pueden encontrar estudios relacionados con el estudio del robo en vivienda (burglary) desde hace varias décadas. 
Hasta hace unos años, gran parte de la información disponible en el Reino Unido sobre el robo en vivienda procedía de datos de la policía, pero a esto se sumó, a últimos de los años 70 , un considerable número de estudios de investigación específicamente ocupados en el robo en vivienda (Walsh 1980); (Maguire and Bennett 1982). (Bennett and Wright 1984) catalogan la motivación de los delincuentes residenciales en seis categorías que abrazan desde las necesidades instrumentales, a la influencia de la presentación de oportunidades, aspecto que puede ligarse a la teoría de actividades rutinarias. El estudio de (Maguire and Bennett 1982), que se hizo conjuntamente con Bennett en el Centro para la Investigación Criminológica de la Universidad de Oxford, ha proporcionado un cuadro especialmente completo sobre el robo en vivienda. Este se basa en la estadística policial de delitos en el área del Valle del Támesis y entrevistas con víctimas y autores de estos tipos de delito conocidos. Los autores analizan, entre otros, el método de entrada al domicilio llegando a la conclusión que en el Reino Unido, la mayor parte de intrusiones se cometen mediante la rotura de ventanas, asimismo, estos autores también se centran en la figura del receptador. Mucho más reciente es la aportación de (Cromwell and Olson 2003) quienes presentan toda una serie de características de los autores, de su motivación por cometer el delito y su particular "iter criminis". Estas características se plantean a través de un estudio cualitativo, basado en una serie de entrevistas a autores de robos con fuerza en vivienda. En el estudio, los autores analizan el proceso racional que utilizan los autores de este tipo de hecho para seleccionar sus objetivos y, a la vez, estudian los efectos de la adicción a las drogas en el modelo de elección racional. El estudio pone de manifiesto la percepción de los riesgos y recompensas relacionadas con la actividad delictiva estudiando de forma empírica la teoría de la elección racional.

En el Reino Unido, el delito del robo en vivienda ha sido estudiado desde múltiples aproximaciones. (Budd 1999) estudia el robo en vivienda desde factores demográficos llegando a la conclusión que, al igual que en el resto de delitos, la gran mayoría de estos son cometidos por hombres y en unas edades comprendidas entre los 16 y los 24 años. A la misma conclusión llega (Mawby 2001). (Webster 2004) estudia el robo en vivienda en relación al origen étnico de los autores llegando a la conclusión que la mayoría de autores de este hecho delictivo son blancos europeos, al igual que el 95\% de las mujeres detenidas. (Farrington, Loeber, and Kalb 2001) se centran en los factores ambientales de 
crecimiento del autor en edad infantil. Los autores llegan a la conclusión que, un ambiente pobre durante la niñez es un considerable factor de riesgo en el desarrollo de tendencias antisociales y en la comisión futura de hechos delictivos como por ejemplo, el robo en vivienda. También en el estudio del robo en vivienda encontramos la aportación de (Hearnden and Magill 2004) quienes, en su trabajo, concluyen que los autores de este hecho delictivo prefieren sustraer dinero, joyas, ordenadores personales y tarjetas de crédito a otros objetos de diverso valor.

También ha habido un interés considerable en la investigación sobre el delito de robo en vivienda en países de Norteamérica. Esto se refleja en los estudios descriptivos de (Scarr 1973) y (Reppetto 1974), autor que analiza las motivaciones de los autores del delito estudiado llegando a la conclusión que la principal motivación, para un asaltante de viviendas, es la satisfacción de su necesidad de dinero. En el Reino Unido, el Home Office publica anualmente el Home Office Statistical Bulletin. Éste boletín sobre los delitos en Inglaterra y Gales presenta los resultados del British Crime Survey y de los datos registrados por la policía del Reino Unido. Esta es una herramienta muy útil para analizar el estado de la cuestión de la totalidad de los delitos ocurridos en el país anglosajón durante el año. Como ejemplo, el último estudio realizado en el año 07/08 muestra que la victimización en el robo en vivienda se ha mantenido igual que en el año anterior, mientras que los datos recogidos por la policía dan como resultado un descenso de un 4\% en este hecho delictivo. Destaca de los resultados del año 2008 el hecho que, sobre un $2 \%$ de las viviendas del Reino Unido habían sufrido un robo o que las viviendas sin medidas de seguridad, como es obvio observar, tenían diez veces más posibilidades de ser robadas que las viviendas con medidas de seguridad simples como una cerradura de seguridad o cerrojos en las ventanas (Kershaw, Nicholas, and Walker 2008).

Las encuestas de victimización nacionales realizadas en los Estados Unidos (Cohen and Cantor 1981) y en Holanda (Van Dijk and Steinmetz 1983) han proporcionado datos muy útiles para el estudio del delito de robo en vivienda. También aporta sus datos de análisis el British Crime Survey elaborado en el Reino Unido que, anualmente, presenta los datos obtenidos sobre la criminalidad en ese país. En este sentido destaca en Cataluña la realización de la Encuesta de Seguridad Pública (en adelante ESPC) con periodicidad anual. La ESPC se inició en el año 1999 por el Departamento de Interior 
del gobierno de la Generalitat de Cataluña, en colaboración con el Instituto de Estadística de Cataluña y la Universidad de Barcelona. La ESPC es una herramienta para la medida y el estudio de la seguridad ciudadana, que sirve para apoyar el diseño, la implantación y la evaluación de las políticas públicas en materia de seguridad.

Según el informe sobre la seguridad en Cataluña del año 2008 elaborado por el Departamento de Interior, Relaciones Institucionales y Participación, la victimización en los delitos cometidos contra las viviendas han experimentado una estabilización, con ligeras fluctuaciones, en el periodo que comprende del año 2000 al 2005. Esta estabilización se rompe en el año 2006 cuando encontramos un sensible aumento aislado, puesto que, en los dos años posteriores, los datos presentan la misma tendencia que durante el inicio de la década. Este ligero aumento en el año 2006 y estabilización posterior, no deja de ser relativamente modesto aunque, en estos tipos de delito, la sensación de inseguridad que genera hace que cualquier variación tenga una repercusión mediática que incrementa todavía más el clima de inseguridad. En materia de hechos contra los domicilios, las diferencias son de décimas y dificultan cualquier conclusión taxativa. A lo largo de todo el periodo, es decir, desde 2000 a 2008, la tendencia ha sido a la estabilización en torno a un 1'7- 1'8, excepto en el año 2006. En este año, la tendencia se interrumpe pasando a un ligero aumento, volviendo rápidamente a índices del inicio del periodo. La tendencia ha sido regular, a excepción del año 2006, y esto, sumado a otras fuentes como las estadísticas policiales, permiten confirmar la existencia de unas ligeras fluctuaciones que no justifican reacciones alarmistas, pero que deben ser tenidas en cuenta. No hace falta añadir que los hechos contra el propio domicilio, aun cuando sólo se produzcan en grado de tentativa, generan inquietud y molestias especialmente intensas.

Estos datos los observamos gráficamente en la siguiente tabla referente a la Encuesta de Seguridad Pública de Cataluña del año 2000 al 2008.

Tabla 1. Porcentaje de personas victimizadas sobre la población total.

\begin{tabular}{|l|llllllllll|l|}
\hline Años & 2000 & 2001 & 2002 & 2003 & 2004 & 2005 & 2006 & 2007 & 2008 \\
\hline Hechos contra la vivenda & 1 1'83 & 1 1'98 & 1 1'64 & 1 1'73 & 1 1'76 & 1 1'91 & 2'13 & 1 '9 & 1'8 \\
\hline
\end{tabular}


A nivel europeo cabe destacar la encuesta de victimización realizada en el año 2004 por parte de Gallup Europe, UNICRI, el Instituto Max Plank, CEPS/INSTEAD y GeoX Ltd. a una muestra de habitantes de la Unión Europea.

Como se ve en este apartado, el estado de la cuestión actual muestra que, en el estado español, ha habido estudios que han venido a mostrar una tendencia a la baja, durante el inicio de la década en los delitos patrimoniales en general y en consecuencia, también en los delitos contra la vivienda.

\section{Metodología}

La investigación que aquí se presenta se ha estructurado de la forma siguiente:

En un primer apartado se estudia la evolución histórica del delito de robo en vivienda en cuatro comarcas. En estas comarcas el despliegue del cuerpo policial de los Mossos d'Esquadra se ha llevado a cabo desde un periodo de tiempo suficiente para observar una evolución significativa, concretamente en las comarcas de: El Gironès (1997), Baix Empordà (1996), Segrià (1999) y La Cerdaña (1997). Estas comarcas se diferencian entre sí por el tipo de residencia (habitual o segunda residencia), tipo de población (autóctona o con un gran índice de inmigración), situación geográfica (montaña, interior, costa). Así pues, se han estudiado comarcas con diferencias substanciales desde el punto de vista de la seguridad ciudadana.

En estas comarcas se ha estudiado en el periodo 2000-2008:

- El número de hechos delictivos contra la vivienda con fuerza.

- El crecimiento del número de viviendas.

- La evolución del paro.

- La evolución de la inmigración, su número y origen.

- Detenidos y su nacionalidad.

- Evolución de efectivos policiales.

Los datos secundarios recogidos son provenientes de bases de datos policiales del cuerpo de los Mossos d'Esquadra y de la policía local, del Departamento de Interior Relaciones Institucionales y Participación. En este trabajo se usan los datos policiales 
obtenidos del Núcleo de Información Policial (NIP), común en la $\mathrm{PGME}^{4}$. Para poder llevar a cabo el objetivo de este estudio se solicitaron al Área de Elaboración de Datos Policiales de la Comisaría General de Planificación y Organización de la Dirección General de la Policía de la Generalitat, los datos referentes a los robos con fuerza en el interior de vivienda y a los detenidos por esta causa.

Los datos referentes a la Policía de la Generalitat de Cataluña (PGME y policías locales conectadas al NIP) han sido extraídos en fecha 8 de Enero de $2009^{5}$, que se establece como fecha de referencia. Al tratarse de un núcleo informático abierto, recibe datos las 24 horas del día, los 365 días del año, y si no se estipulara una fecha de referencia, los datos sufrirían variaciones constantes que dificultarían su estudio.

Otros datos estadísticos oficiales que han sido estudiados son los siguientes:

- Instituto de Estadística de Cataluña. En las estadísticas extraídas de esta fuente se debe tener presente que los datos sobre la población aportadas por el Instituto de Estadística de Cataluña son datos oficiales y por lo tanto no recogen los datos sobre población no empadronada. Los datos sobre población son los datos del 1 de enero de cada año.

- Departamento de Medio Ambiente y Vivienda de la Generalitat de Cataluña.

- Encuesta de Seguridad Pública de Cataluña.

- Departamento de Interior, Relaciones Institucionales y Participación.

- Ministerio del Interior.

Para la obtención de los datos del Núcleo de Información Policial se ha utilizado el programa informático Datawarehouse de la firma MicroStrategy en su versión 8.0 .2

\footnotetext{
4 Los datos de este trabajo recogen la información de 144 policías locales conectadas al núcleo de información policial, los municipios de las cuales tienen firmado el convenio con el Departamento de Interior, Relaciones Institucionales y Participación para la introducción de datos policiales.

${ }^{5}$ Dado que el núcleo de información policial (NIP) de la PGME es un instrumento dinámico, sus estadísticas se actualizan permanentemente. Por este motivo se hace necesario incorporar una fecha de referencia para oficializar unos datos únicos tanto interna como externamente, al efecto de facilitar la extracción y análisis con criterios homogéneos.
} 


\section{Estudio de las diversas variables.}

\subsection{Crecimiento demográfico:}

A nivel demográfico, en las cuatro comarcas estudiadas, el crecimiento experimentado ha sido prácticamente a causa del movimiento migratorio de personas con origen en otros países.

Tabla 2. Crecimiento demográfico en Cataluña.

Fuente: Elaboración propia con datos del Instituto de Estadística de Cataluña.

\begin{tabular}{|llllllllll|}
\hline Años & 2000 & 2001 & 2002 & 2003 & 2004 & 2005 & 2006 & 2007 & 2008 \\
\hline Cataluña & 6.261 .999 & 6.361 .365 & 6.506 .440 & 6.704 .146 & 6.813 .319 & 6.995 .206 & 7.134 .697 & 7.210 .508 & 7.364 .078 \\
\hline Inmigración & 181.590 & 257.320 & 382.020 & 543.008 & 642.846 & 798.904 & 913.757 & 972.507 & 1.103 .790 \\
\hline
\end{tabular}

Como se observa (Tabla 2), la población de Cataluña ha aumentado en el periodo estudiado en 1.100.000 habitantes, de los cuales 922.000 corresponden a ciudadanos provenientes de otros países. Así pues, prácticamente el crecimiento observado en este periodo se corresponde a un aumento de población inmigrada.

\subsection{Inmigración:}

En la tendencia que describe este crecimiento encontramos ciertas peculiaridades dependiendo de cada comarca en el periodo analizado. En alguna comarca, como el caso del Gironès, encontramos una diferencia respecto del resto de las comarcas y es que se observa una disminución en el crecimiento del número de inmigrantes en el año 2001. Este hecho, que sólo se encuentra en aquel año y en aquella comarca, da lugar a poder interpretar que en aquel año se encontró un problema en la recogida de datos. Las cuatro comarcas tienen en común un aumento regular en el número de personas inmigradas, con repuntes en este aumento, en los años 2005 y 2008. Estos datos también se relacionan con el crecimiento demográfico de Cataluña en cuanto a población inmigrada, puesto que, en los años 2005 y 2008 son los que el crecimiento migratorio fue más grande. Las comarcas estudiadas, pues, presentan un paralelismo en Cataluña y podemos inferir que la inmigración llegada a esta comunidad se ha repartido por todo el territorio no solo en algunas zonas. Cabe destacar que en algunas zonas, como las capitales provinciales y en las zonas productivas, su número ha sido mayor. Este reparto 
de la inmigración por las diferentes comarcas de Cataluña no ha sido regular en cuanto a la nacionalidad de las personas recién llegadas. Del estudio de los datos se extrae que, los individuos inmigrados tienen tendencia a agruparse en zonas concretas dando lugar a concentraciones de nacionalidades en unas comarcas y la ausencia o presencia mínima en otras ${ }^{6}$. Es en la distribución por nacionalidades dónde las diferencias comarcales son más destacables.

En la comarca del Segrià hay dos nacionalidades predominantes que son la rumana y la marroquí, esta última había sido la más numerosa hasta el año 2007. En ese año, se vio superada por la nacionalidad rumana que, en esta comarca, ha tenido un crecimiento muy significativo desde el año 2003. El resto de nacionalidades están muy lejos en número de estas dos. Por otra parte, esta distribución no esta presente en el resto de comarcas estudiadas.

En la comarca del Gironès, la nacionalidad predominante es la marroquí y con mucha diferencia del resto de nacionalidades que presentan un crecimiento más suave. En esta comarca destaca el segundo lugar de personas originarias de Honduras. También encontramos que los inmigrantes originarios de Marruecos son los más numerosos de la comarca del Baix Empordà y, al igual que en la comarca anterior, hay una gran diferencia con el resto de nacionalidades que presentan un crecimiento muy sostenido. Por último, en la comarca de la Cerdaña la nacionalidad que presenta un mayor número de ciudadanos es la boliviana que presenta un crecimiento muy superior al resto y con una diferencia notable de los ciudadanos portugueses que son los que ocupan el segundo lugar.

Así pues, del análisis demográfico anterior se extrae que, dentro de la población inmigrada, en las comarcas estudiadas hay un predominio de una nacionalidad, principalmente la marroquí. Existe la excepción de la comarca del Segrià dónde este predominio está compartido entre dos nacionalidades, la marroquí y la rumana. Esta distribución está relacionada con la de Cataluña, puesto que en el año 2008 la nacionalidad más numerosa era la marroquí, con un $18,94 \%$ de la población extranjera y la rumana, con un 7,98\%. Así es destacable la concentración de ciudadanos rumanos en la comarca del Segrià.

\footnotetext{
${ }^{6}$ En este sentido, (García España 2001).
} 


\subsection{Desempleo en Cataluña y comarcas:}

\section{Gráfico 1. Desempleo en Cataluña.}

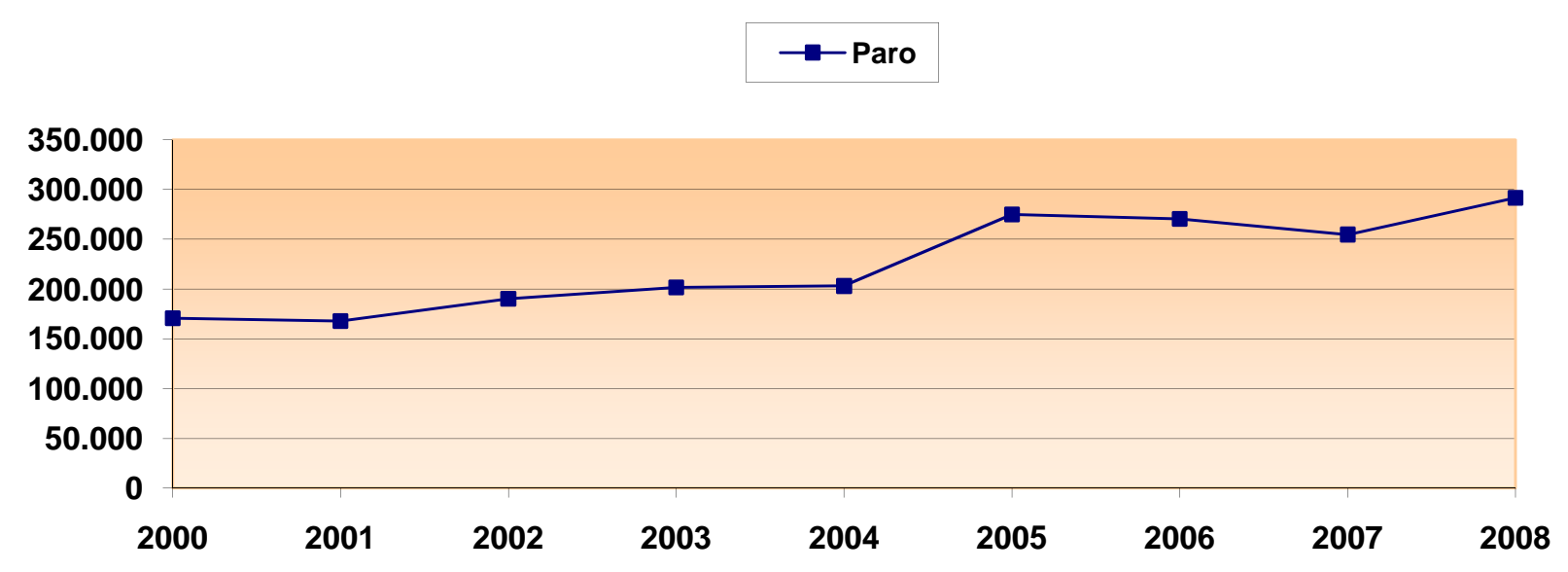

Gráfico 2. Desempleo en la cuatro comarcas analizadas.

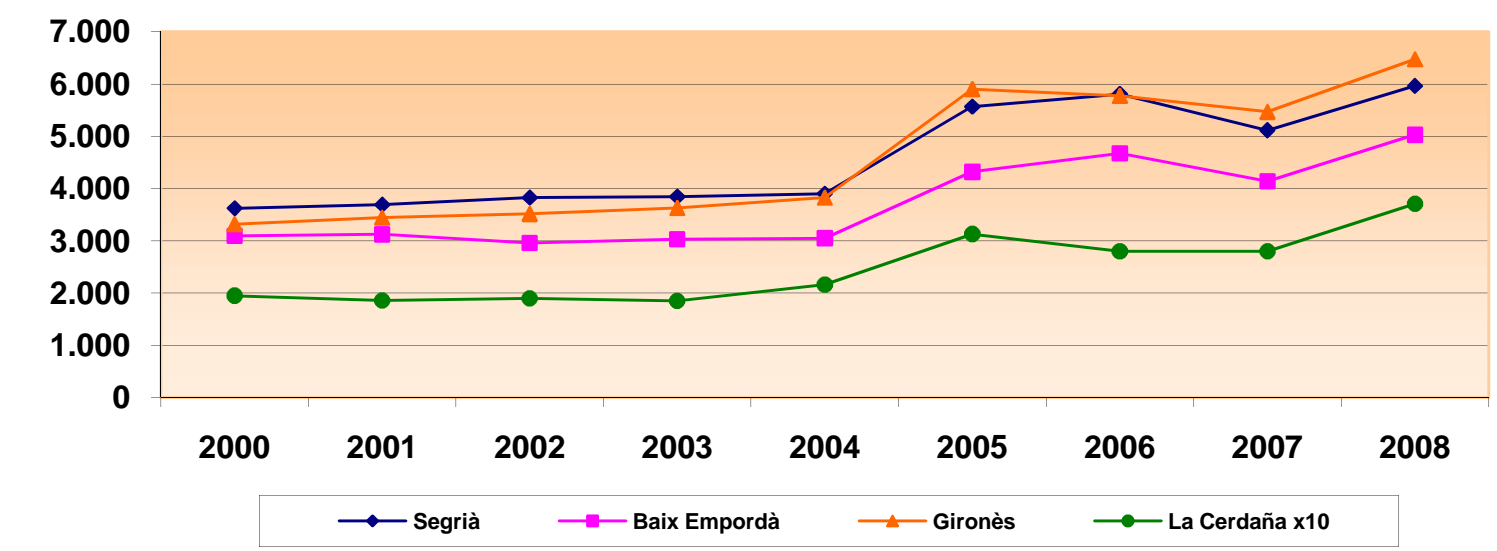

Fuente: Elaboración propia con datos del Instituto de Estadística de Cataluña.

En cuanto al paro (gráficos 3 y 4), tras analizar los datos, destaca que durante los años del inicio de la década del 2000 podemos hablar de prácticamente plena ocupación en las comarcas estudiadas. Se inicia un aumento en el número de desempleados, con altibajos, durante el periodo estudiado con dos momentos diferenciados en su tendencia al alza, uno es el año 2005 y el otro en el año 2008. En estos dos años se pasa en todas las comarcas de un aumento o descenso, con unas oscilaciones suaves, a un aumento del paro muy significativo en el año 2005, una estabilización posterior y otro significativo aumento en el año 2008. Este patrón de conducta del paro, como se ha dicho, esta presente en mayor o menor medida en las comarcas analizadas y también es un reflejo de la situación global de Cataluña. Así pues, encontramos una correlación entre un 
fuerte aumento de la inmigración los años 2005 y 2008 y un fuerte aumento del paro en los mismos años. Este aumento en el número de desempleados es, en gran medida causado por la caída del sector de la construcción, sector que hasta hace pocos años era capaz de absorber el creciente número de población de las comarcas analizadas.

\subsection{Construcción de viviendas en las comarcas estudiadas:}

Gráfico 3. Viviendas iniciadas en las cuatro comarcas.

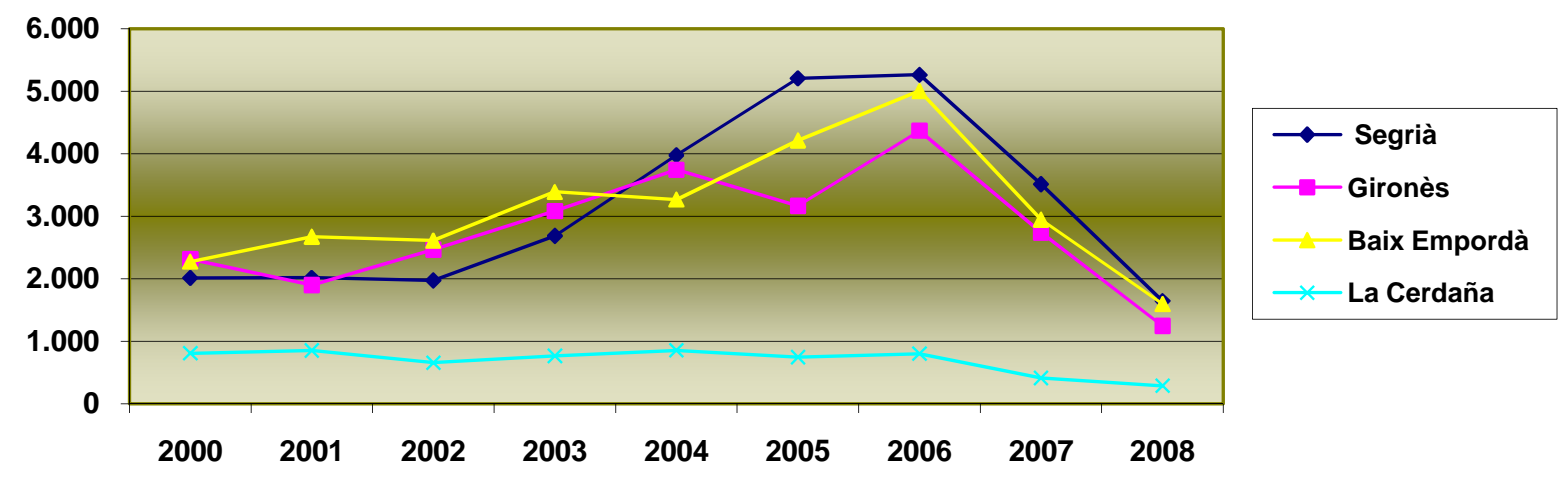

Fuente: Elaboración propia con datos del Departamento de Medio Ambiente y Vivienda de la Generalitat de Catalunya.

En referencia a la construcción de viviendas, en las cuatro comarcas estudiadas se observan comportamientos diferentes. De una parte encontramos que, en la comarca del Segrià es dónde el inicio de la construcción de viviendas (gráfico 3) presenta un aumento más significativo a partir del año 2002. En este año, se inicia una fuerte actividad constructora hasta llegar al año 2005, en el que se estabiliza y cae a partir del año 2006. Este comportamiento no se observa en el resto de comarcas, como en la Cerdaña debido a su actividad constructora con tendencia a la segunda residencia. Esta comarca presenta altibajos habituales en una comarca turística. Esta misma dinámica se observa en las comarcas del Gironès y del Baix Empordà dónde la actividad constructora presenta un significativo aumento pero con años de descenso en dicha actividad. El que sí es común en todas las comarcas es una fuerte disminución en el número de viviendas iniciadas a partir del año 2006. 


\section{Gráfico 4. Viviendas finalizadas en las cuatro comarcas.}

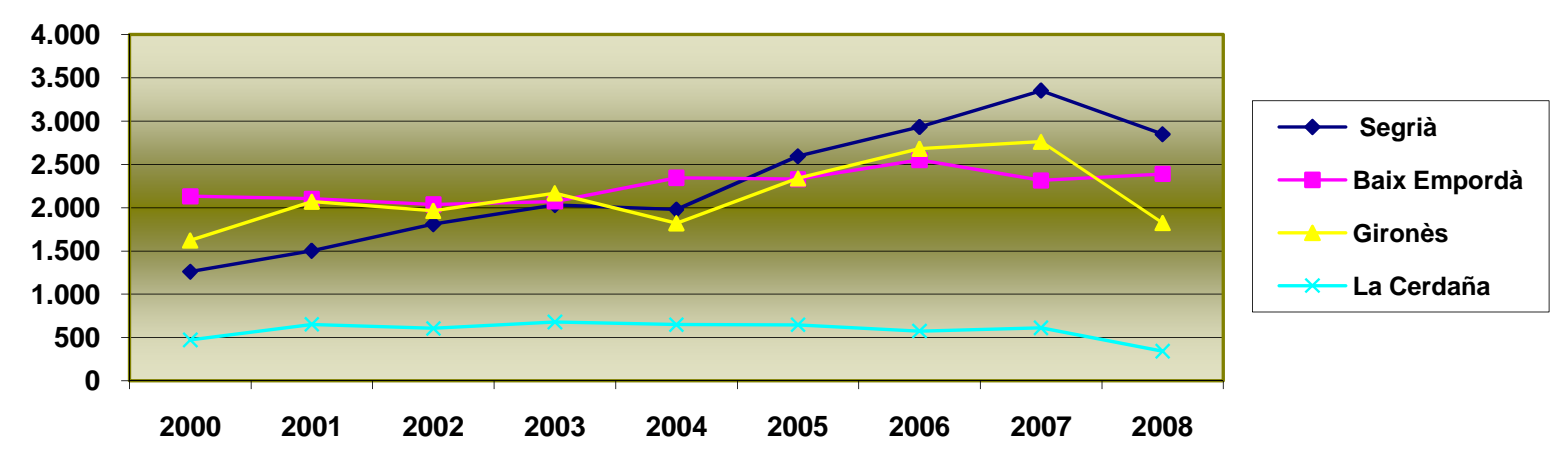

Fuente: Elaboración propia con datos del Departamento de Medio Ambiente y Vivienda de la Generalitat de Catalunya.

En el número de viviendas acabadas (gráfico 4) no se observan las oscilaciones que se podían observar en los edificios iniciados, puesto que, la tendencia es mucho más regular. En cuanto a viviendas acabadas, el Segrià presenta el mayor aumento durante los últimos años. En el resto, la finalización sigue unos parámetros similares a los del inicio de viviendas, a excepción del Gironès, dónde en los años 2001 y 2004 su tendencia es a la inversa. De los datos observados en las comarcas estudiadas se extrae que, durante este periodo analizado, ha aumentado de forma notable la oferta de viviendas al mercado inmobiliario. El número de domicilios o viviendas capaces de albergar familias o residentes ha aumentado de forma muy significativa. Podemos decir que esta mayor oferta de vivienda no ha correlacionado en una mayor comisión de delitos contra el domicilio. Contrariamente, durante el periodo en que ha habido un crecimiento en el inicio y la finalización de la construcción de viviendas, se ha observado en general una bajada o estabilización en este tipo de delito. Así pues, la construcción de viviendas no parece influenciar en la comisión de este hecho delictivo. 


\subsection{Distribución de efectivos policiales:}

\section{Gráfico 5. Distribución de efectivos policiales en las cuatro comarcas.}

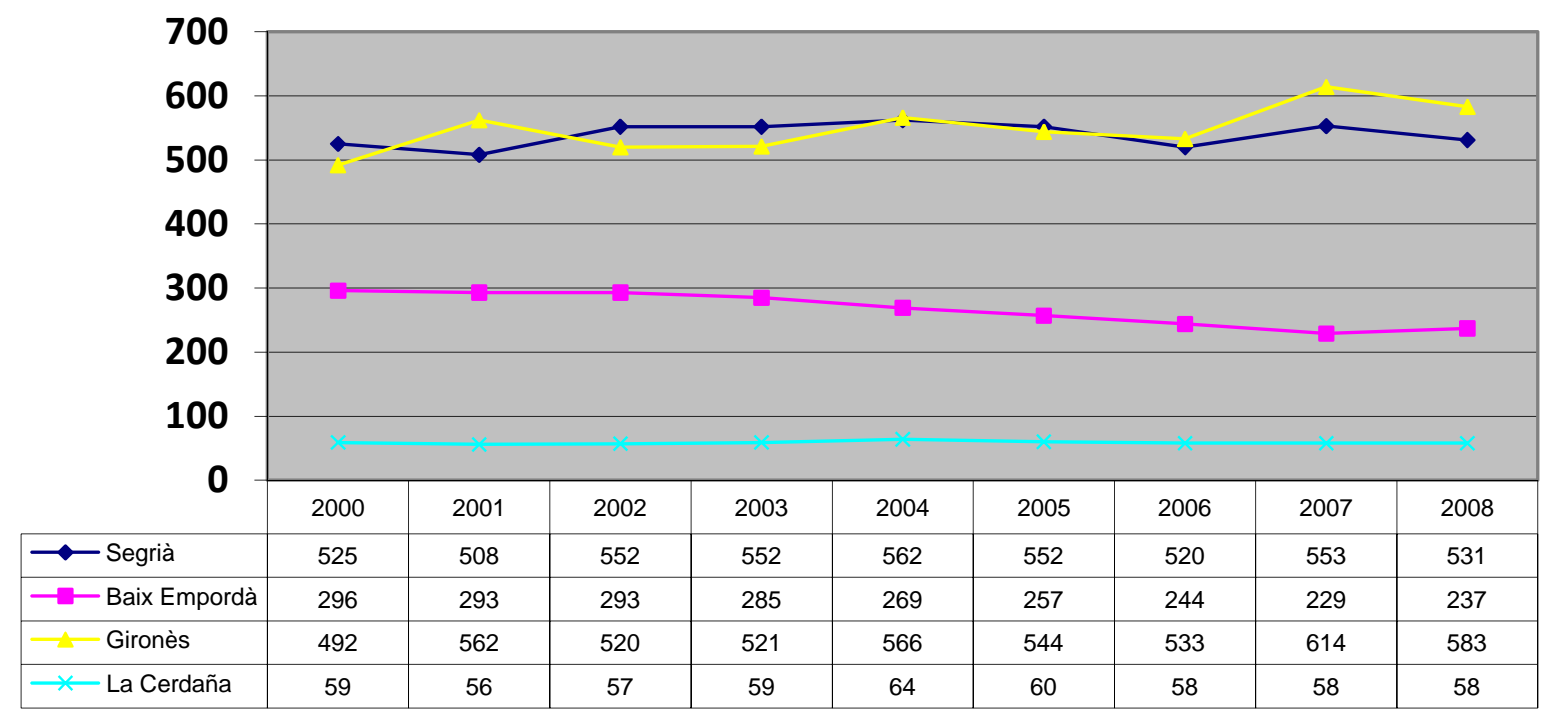

Fuente: Elaboración propia con datos del Instituto de Estadística de Cataluña.

En los datos referentes al número de efectivos policiales (gráfico 5) observamos que las cuatro comarcas tienen en común un mantenimiento o, en ocasiones, descenso en el número de efectivos. La comarca del Segrià y la de la Cerdaña son las comarcas que presentan mayor irregularidad en la distribución anual de su número de efectivos. Por otro lado, las comarcas del Baix Empordà y Gironès son las que presentan una mayor regularidad en su distribución. En este periodo estudiado se identifica la importancia del despliegue del cuerpo policial de los Mossos d'Esquadra en cuanto a la evolución de este tipo de hecho delictivo. La presencia policial en el territorio es fundamental a la hora de prevenir y responder a la actividad criminal. La presencia de efectivos policiales en una zona del territorio puede influir en gran manera en la distribución de hechos delictivos, a la vez que, una fuerte presencia en otra zona geográfica puede comportar una disminución de hechos puesto que esta presión policial puede disuadir a ciertos individuos a cometer ilícitos o a desplazarse a otras áreas geográficas. No se trataría de un desplazamiento de la delincuencia, sino de una parte de la delincuencia, puesto que, el resto tiende a disminuir la intensidad de su carrera delictiva. Este fenómeno lo 
podemos observar en el siguiente gráfico que muestra la actividad delictiva de Barcelona en los años 2006, 2007 y 2008.

\section{Gráfico 6. Robos con fuerza en domicilio en la ciudad de Barcelona, años 2006-}

\section{8}

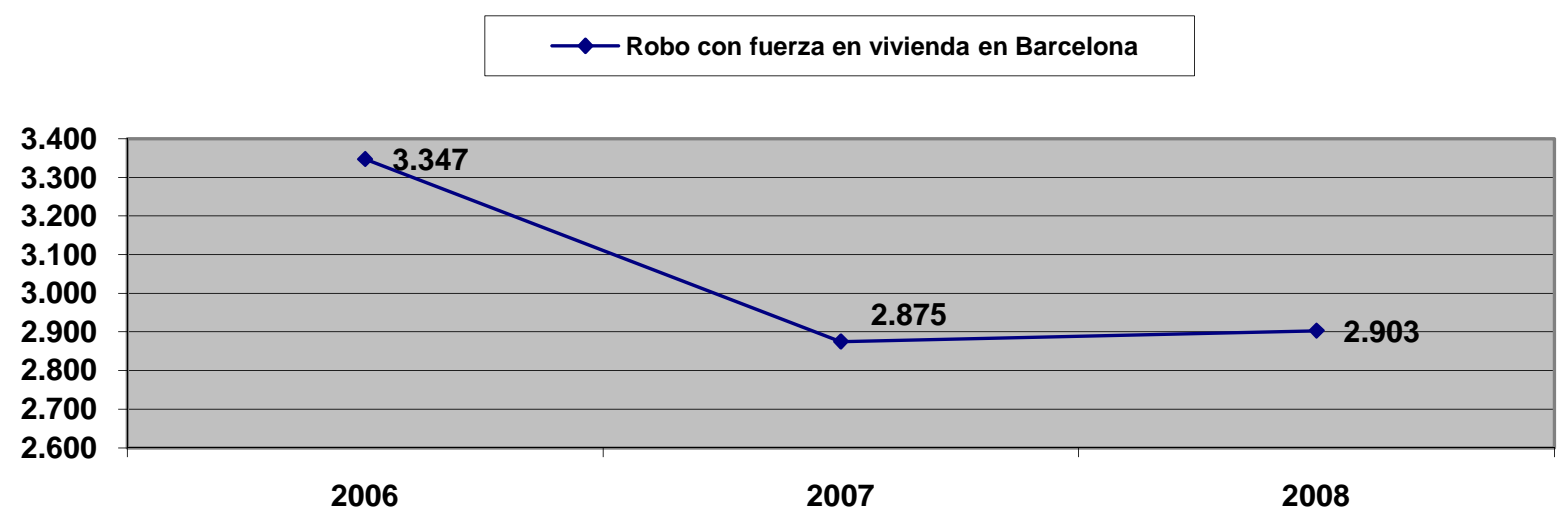

Fuente: Elaboración propia con datos de la Dirección General de la Policía del Departamento de Interior y Relaciones Institucionales de la Generalitat de Catalunya.

Mientras que en el resto de comarcas, en estos últimos años, se observa un repunte en el número de hechos delictivos, en esta ciudad se ha reducido y estabilizado (gráfico 6). Esta circunstancia se puede dar, entre otras, por el hecho del inicio del despliegue del cuerpo de Mossos d'esquadra en Barcelona, en noviembre de 2005, momento en que hay un importante despliegue de efectivos que no sólo llevan a cabo tareas de prevención sino de disuasión con controles de carretera o de investigación.

Esta mayor presencia de efectivos en las zonas del área metropolitana y la mejora de la red de comunicaciones por carretera, como la apertura de la C-25 y la autovía A-2 entre Lleida y Barcelona, ha comportado que la presión ejercida en las comarcas de nuevo despliegue haya favorecido un desplazamiento de ciertos individuos muy activos, en este tipo de hecho delictivo, hacia comarcas con menos presencia policial. No podemos hablar de un desplazamiento de la delincuencia hacia otras zonas, pero si podemos exponer que una fuerte presión en el área metropolitana, puede desplazar a ciertos grupos especializados en el robo en viviendas a otras zonas geográficas dónde el control formal sea menos intenso. 


\subsection{Robos con fuerza en vivienda:}

\section{Gráfico 7. Robo con fuerza en vivienda en las cuatro comarcas.}

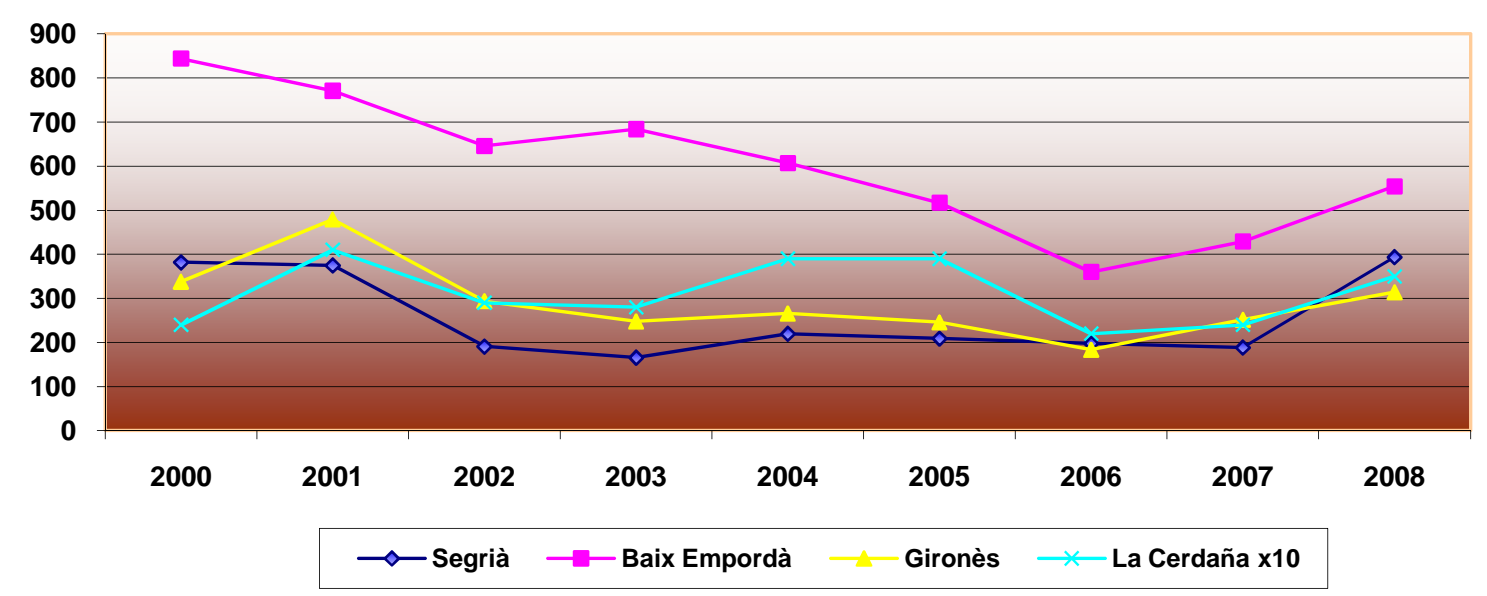

Fuente: Elaboración propia con datos de la Dirección General de la Policía del Departamento de Interior i relaciones institucionales de la Generalitat de Catalunya.

$\mathrm{Si}$ analizamos los datos referentes al delito de robo con fuerza en interior de vivienda (gráfico 7) podemos extraer las valoraciones siguientes: En las cuatro comarcas estudiadas se partía de un elevado número de delitos al inicio del periodo, principalmente en los años 2000 y 2001. A continuación, las tres comarcas con más población experimentan una progresiva disminución de hechos delictivos. En este sentido, la comarca que presenta una tendencia más regular es la comarca del Baix Empordà, puesto que, se aprecia un descenso constante en el número de hechos conocidos. Éste descenso se produce de forma suave hasta llegar al año 2006, en el que los hechos conocidos son los más bajos e iniciando posteriormente un aumento también regular en el registro de ilícitos. Este comportamiento también se ve claramente en la comarca del Gironès, dónde el descenso de hechos conocidos es más irregular pero también se llega al año 2006 con el menor número de hechos. Acto seguido se inicia una recuperación de delitos que se mantiene durante el año 2008. La comarca de la Cerdaña también presenta esta tendencia aunque aquí las oscilaciones de hechos conocidos son más bruscas pero, al llegar al año 2006, imita el comportamiento de las otras dos comarcas, empezando a aumentar el número de hechos conocidos. La comarca del Segrià se ha dejado para el último lugar, puesto que, en esta comarca la distribución de hechos delictivos ha sido la más atípica. En esta comarca encontramos un alto 
número de hechos delictivos los años 2000 y 2001 que caen de forma brusca a prácticamente la mitad en el año 2002. El número de hechos conocidos se mantiene estable durante un periodo de seis años y, de la misma forma brusca del año 2002, en el año 2008 se observa un aumento de hechos conocidos que duplica el número de hechos del año anterior. En esta comarca el aumento, que habían sufrido las otras comarcas y que se había visto reflejado el año 2007, se ha producido directamente en el año 2008. Podemos decir que la tendencia a la baja en este hecho delictivo lo hemos visto en todas las comarcas analizadas aunque no de la misma forma en todas ellas, puesto que, en algunas ha estado de forma más irregular.

\section{Gráfico 8. Índice de criminalidad robo con fuerza/1.000 habitantes.}

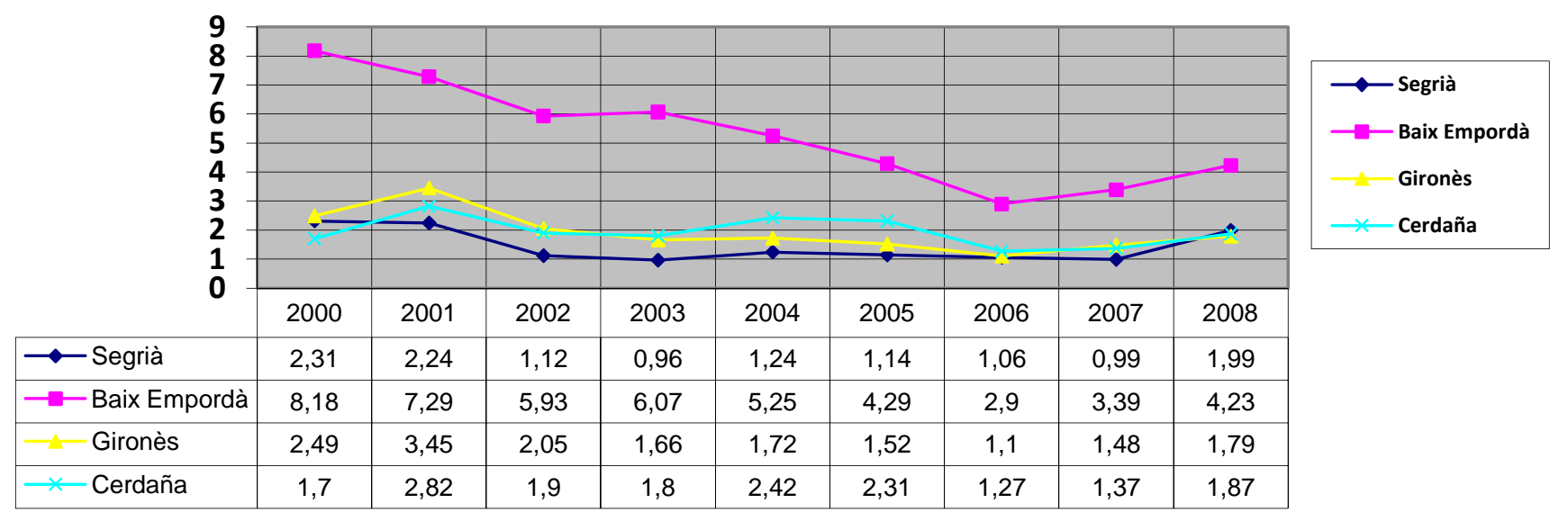

Fuente: Elaboración propia con datos de la Dirección General de la Policía del Departamento de Interior i relaciones institucionales de la Generalitat de Catalunya.

En cuanto a la incidencia de este hecho delictivo por 1.000 habitantes (gráfico 8) se observa que el índice de criminalidad más alto, en cuanto al delito estudiado, corresponde a la comarca del Baix Empordà. Esta comarca presenta unas ratios que superan con creces más del doble de las ratios de las otras comarcas, aun cuando se debe tener en cuenta que a la hora de cuantificar metodológicamente las viviendas pueda haber alguna disfunción, ya que, algunas segundas residencias pueden cuantificarse como vivienda principal, parece una ratio elevada en comparación con el resto. Estos índices experimentan un descenso hasta el año 2006, cuando inician un aumento significativo, al igual que los hechos conocidos. Se puede destacar en esta 
comarca que, aunque sea la que presenta unos índices más elevados, ha pasado de un índice de más de un 8\% del año 2000, a la mitad en el año 2008. En cuanto al resto de comarcas, la comarca del Gironès presenta, al inicio del periodo, sus índices más elevados que muestran un claro descenso. Se observa una suave recuperación a partir del año 2006 pero que, en ningún caso, llegan a los índices del inicio del periodo analizado y $\sin$ sobrepasar un $2 \%$ el año 2008 . Un comportamiento muy similar al del Gironès lo encontramos en la comarca de la Cerdaña. En esta comarca se observan unos índices que superan tres veces el 2\% y que acaban, en el año 2008, con un índice prácticamente igual al de la comarca del Gironès. En la comarca del Segrià encontramos unos índices similares a los de las comarcas del Gironès y de la Cerdaña, pero todavía más bajos. El aumento de hechos delictivos del último año 2008 ha hecho que el índice del año 2008 se acercara al de los años 2000 y 2001. El hecho que los índices de los años anteriores fueran muy bajos ha hecho que, todo y la subida del índice, no se llegara al 2\%o superando pues, por muy poco, a las comarcas de la Cerdaña y el Gironès y siendo menos de la mitad del de la comarca del Baix Empordà. El aumento de hechos delictivos denunciados en los últimos años ha sido amortiguado por el aumento de población a la hora de valorar los índices de criminalidad en el hecho delictivo analizado. En el grafico anterior se ve la tendencia similar en los índices de criminalidad del delito de robo con fuerza en vivienda. La comarca del Baix Empordà muestra similar tendencia que el resto pero siempre con unos índices mas elevados.

\subsection{Detenidos por nacionalidad:}

Gráfico 9. Detenidos por robo con fuerza por nacionalidad en la comarca del Segrià.

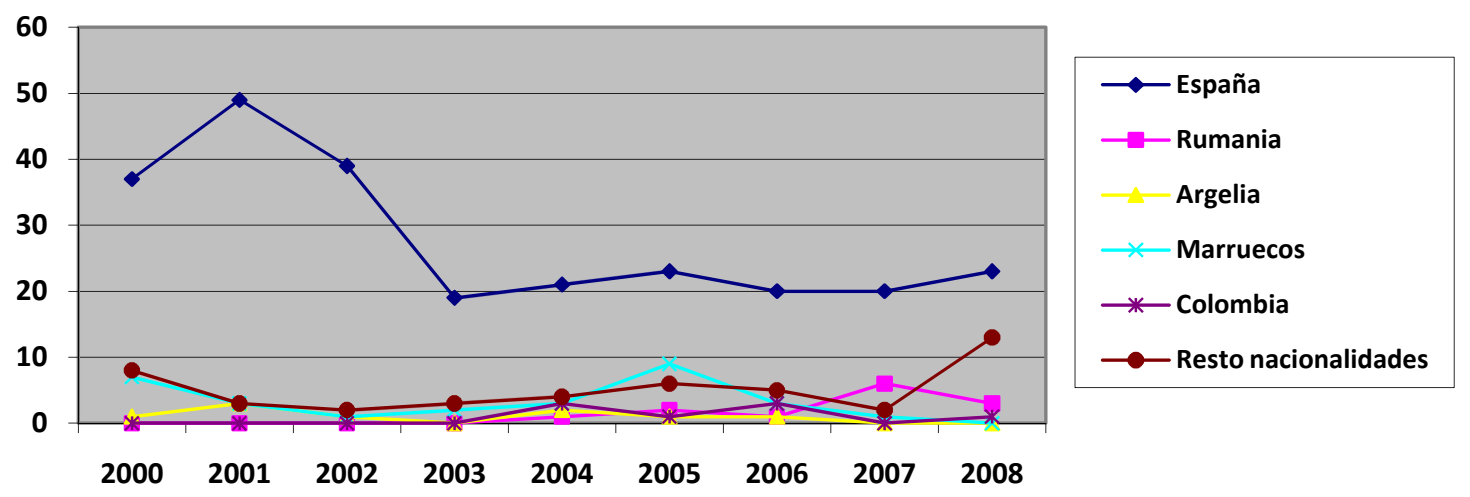


Gráfico 10. Detenidos robo con fuerza por nacionalidad en la comarca del Baix Empordà

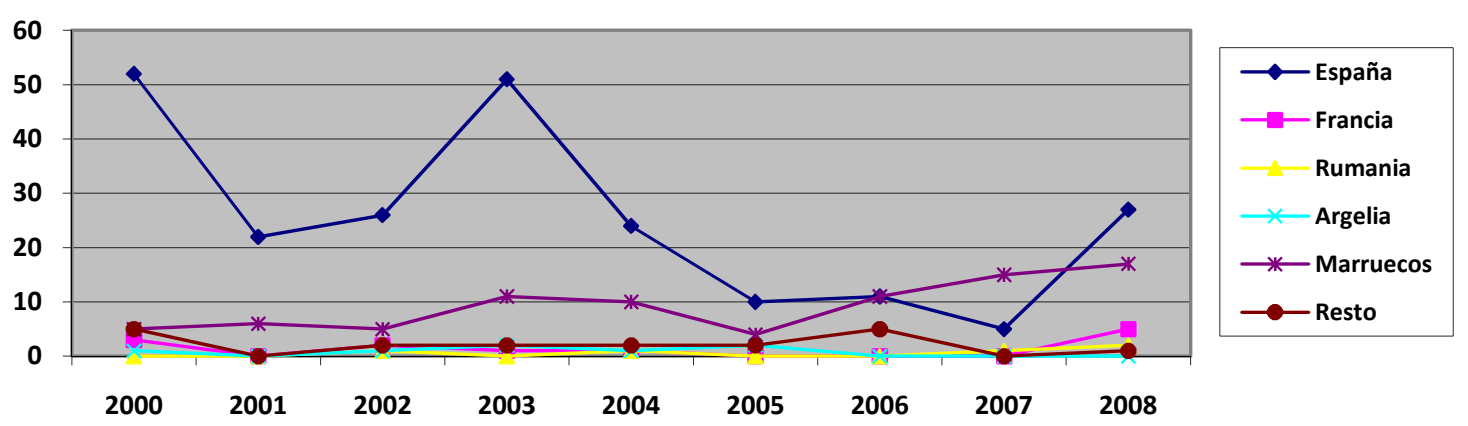

\section{Gráfico 11. Detenidos robo con fuerza por nacionalidad en la comarca del Gironès}

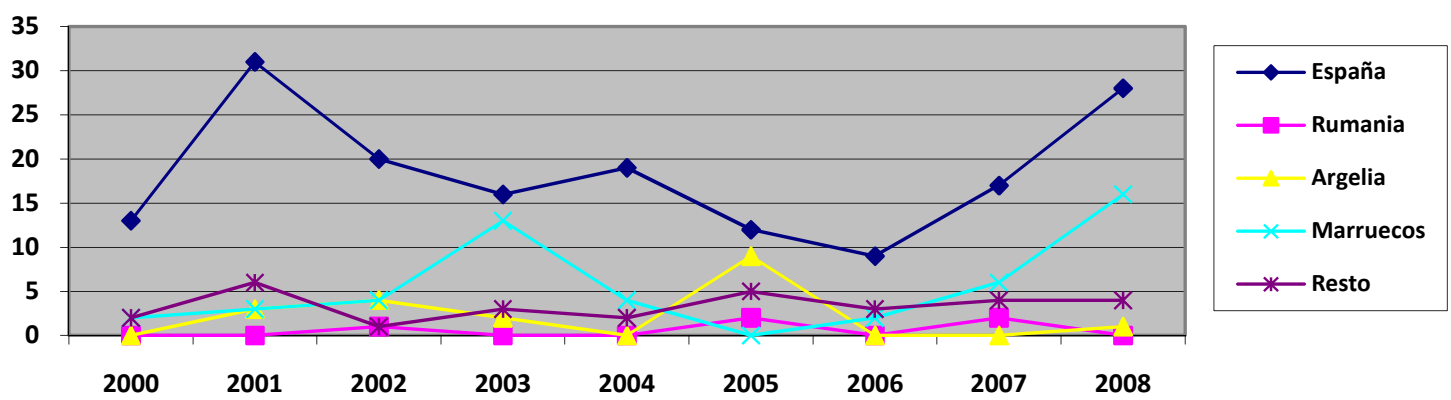

Fuente: Elaboración propia con datos de la Dirección General de la Policía del Departamento de Interior i Relaciones Institucionales de la Generalitat de Catalunya.

En cuanto a la valoración del número de detenidos por el delito de robo con fuerza en vivienda (gráficos 9,10 y 11) encontramos que hay diferencias significativas, si comparamos una comarca con otra. Observamos por ejemplo, que en la comarca del Baix Empordà hay un paralelismo entre la disminución de hechos delictivos y la disminución de detenciones por este tipo de delito en la población más numerosa, que es obviamente la española. En la comarca encontramos un gran número de detenidos al inicio del periodo, un descenso pronunciado los dos años posteriores y otro aumento que nos trae a datos del inicio de la década. Es a partir del año 2003 que nos encontramos con un notable descenso de detenidos pasando, el año 2004, a la mitad de los del año anterior y un año 2003, con la mitad de los del 2004. Este descenso se mantiene con su nivel más bajo el 2007 cuando se rompe esta tendencia y se pasa a un número de detenidos similar a los del año 2002, es decir, el año 2008 se quintuplican los del año anterior. Este aumento de detenciones se corresponde con un aumento de delitos en este año 2008. Si analizamos el número de detenidos por la nacionalidad más 
numerosa, excluyendo la nacionalidad española, nos encontramos que al igual que el reparto demográfico, el mayor número de detenidos corresponde a la nacionalidad marroquí. En esta comarca las nacionalidades más numerosas son las que más detenidos aportan a este hecho delictivo. Los detenidos de nacionalidad marroquí habían aumentado hasta el año 2004, cayendo bruscamente el año 2005 recuperándose y continuando una progresión ascendente hasta el año 2008. Destaca el hecho que, en el año 2007, hubo más detenidos de origen marroquí que de nacionalidad española, tendencia que se había generado el año anterior y que se ha visto rota por el fuerte aumento de detenidos de nacionalidad española del año 2008. En la comarca del Gironès la distribución de los detenidos por nacionalidad es más irregular, puesto que presenta unas oscilaciones más marcadas. La nacionalidad española, al igual que en las otras comarcas, es la que más detenidos presenta. Al inicio del periodo encontramos un numero elevado de detenidos que no presenta una tendencia clara durante el periodo analizado, puesto que, hay aumentos y descensos en el número de detenidos de un año a otro. El que si que tiene en común esta comarca con la del Baix Empordà es un aumento de detenidos a partir del año 2006. En esta comarca, la segunda nacionalidad en número de detenidos, al igual que en la comarca anterior, es la marroquí. Esta nacionalidad, al igual que la española, presenta un aumento en el número de detenidos a partir del año 2005, año en el que se detuvo a un menor número de ciudadanos de origen marroquí. En este caso también se corresponde el hecho que, el mayor número de detenidos se corresponde con el mayor número de ciudadanos en la comarca. En la comarca de la Cerdaña, el bajo número de detenciones no permite obtener una tendencia en el número de detenidos, el mayor número de detenidos son de nacionalidad española y contrariamente a lo que se observa en otras comarcas el mayor número los encontramos en el año 2007. Durante el año 2008 encontramos una disminución significativa pero, como se ha dicho anteriormente, el bajo número de detenciones no permite extraer unas características únicas. También podemos destacar que aunque en la comarca, la segunda nacionalidad más numerosa es la boliviana, no encontramos ningún detenido de esta nacionalidad, por este hecho delictivo, durante el periodo analizado.

Finalmente, en la comarca del Segrià, encontramos un gran número de detenidos de nacionalidad española, al inicio del periodo estudiado, y podemos observar un descenso importante hasta el año 2003 en el que se inicia un periodo de estabilización que 
permanece durante el año 2008. Podemos observar que la comarca del Segrià es la única que no presenta un aumento de detenidos de nacionalidad española en el año 2008. Se observa un aumento de detenciones, de nacionales de países sin representación en las 5 nacionalidades más numerosas. En esta comarca también se ve reflejado el hecho que las nacionalidades más numerosas, fuera de la nacionalidad española, también son las más numerosas en número de detenciones, (Marruecos y Rumania). El fuerte aumento de inmigrantes de nacionalidad rumana, durante los últimos años en la comarca, muestra un paralelismo con las detenciones practicadas en el periodo analizado.

\section{Conclusiones}

En primer lugar, el crecimiento demográfico experimentado en Cataluña ha sido prácticamente debido a la inmigración, puesto que, en el periodo analizado, se observa que el crecimiento total coincide en gran manera con el crecimiento del número de personas inmigradas. En este periodo, Cataluña ha incrementado su población en 1.102.000 personas, de las cuales, 922.000 son de origen extranjero. Además, se debe tener presente que, aparte de las cifras oficiales, hay un gran número de personas de origen extranjero en situación irregular que no están contabilizadas por no constar en el padrón municipal. Un importante factor de riesgo, para delinquir, es la situación de ilegalidad en la que se puede encontrar un individuo inmigrante (García España 2001).

Este crecimiento demográfico de origen extranjero, experimentado en las comarcas estudiadas, no ha comportado un aumento en los índices de criminalidad en referencia al delito de robo en vivienda. Como se ha expuesto anteriormente, mientras aumentaba la población disminuían los hechos delictivos. Este aumento de población extranjera ha sido absorbido por el sector productivo, puesto que, sólo se han observado aumentos del paro en periodos de gran llegada de población extranjera.

En segundo lugar, se observa una disminución de detenidos de nacionalidad española y un aumento de detenidos de nacionalidad marroquí y rumana. Podemos hablar de una sustitución en la autoría de estos hechos delictivos. Ciertos individuos, de comunidades de inmigrantes con un mayor número de ciudadanos, están sustituyendo a una parte de autores de estos tipos delictivos de nacionalidad española como consecuencia de un proceso de sustitución en la escala social. En tercer lugar, el aumento del paro no sólo en el sector de la construcción, puede dar lugar a un aumento de hechos delictivos 
contra la vivienda, como se ha constatado a partir del año 2007, coincidiendo con un notable repunte en este tipo de hechos delictivos. En los últimos años se debía destacar una notable ocupación, que a partir del año 2006 pasa a mostrar un aumento de desocupación. Es esta desocupación la que puede traer, en casos puntuales, a la marginalidad y a un incremento de actividades delictivas contra la vivienda. Sobretodo, en individuos que no puedan satisfacer sus expectativas o la influencia de otros les pueda empujar a una carrera criminal abandonada durante el periodo de bonanza económica, con unos sueldos relativamente altos, para una formación profesional limitada.

Los datos analizados en este estudio vienen a mostrar que, las comarcas observadas tienen un índice de criminalidad en el delito de robo con fuerza en vivienda por debajo de la media española. La única comarca que está por encima de la media es la comarca del Baix Empordà pero ligeramente por encima (España 3’71\%o, Baix Empordà. 4\%o). La media catalana en el año 2007 fue de un 4’02 \%o.

El aumento demográfico que ha experimentado Cataluña y sus comarcas en general, debería corresponderse con un aumento de efectivos policiales, puesto que, en la mayoría de las comarcas analizadas, las dotaciones policiales no han aumentado en la misma proporción que el aumento de población. Un aumento en el número de efectivos policiales distribuidos territorialmente muestra una gran efectividad, como se ha mostrado. En las comarcas dónde el numero de efectivos ha aumentado o se ha mantenido de forma regular, el incremento de hechos delictivos ha aumentado de forma sostenida. Por lo tanto, se confirma que un aumento o disminución de efectivos policiales puede favorecer una mayor o menor comisión de estos hechos delictivos.

Por último, en este tipo de delito actúan, a menudo, grupos especializados, no delincuencia organizada, que se desplazan por el territorio ya sea a nivel comarcal, autonómico o estatal, por tal de cometer un gran número de hechos delictivos en poco espacio de tiempo. Este tipo de autoría propicia que con pocas detenciones, la imputación de hechos delictivos sea elevada, así pues, con la detención de un individuo se pueden resolver múltiples hechos. 


\section{Referencias}

"Balance.", Retrieved 04/11/2009, 2009. (http://www.mir.es/DGRIS/Balances/Balance_2009/pdf/Bal_criminalidad_julio_20 08 junio_2009).

Avilés Ferré, J. 2003. "La Delincuencia En España: Una Aproximación Histórica (19502001)." Historia Del Presente 2:125-138.

Bennett, T. and R. Wright. 1984. Burglars on Burglary: Prevention and the Offender. Gower Aldershot, Hants.

Budd, T. 1999. "Burglary of Domestic Dwellings: Findings from the British Crime Survey." No 4:99.

Cohen, L. E. and D. Cantor. 1981. "Residential Burglary in the United States: Life-Style and Demographic Factors Associated with the Probability of Victimization." Journal of Research in Crime and Delinquency 18(1):113.

Cromwell, P. F. and J. Olson. 2003. Breaking and Entering: Burglars on Burglary. Wadsworth Publishing Company.

Farrington, DP, R. Loeber and LM Kalb. 2001. "Key Research and Policy Issues." Child Delinquents: Development, Intervention, and Service Needs.Thousand Oaks, CA: Sage Publications:385-94.

Fernández-Ramírez, B. 2008. "Seis Hipótesis De Trabajo Para Entender La Delincuencia y El Miedo Al Delito." Revista Española De Investigación Criminológica [En Línea] 6.

García España, E. 2001. "Inmigración y Delincuencia En España: Análisis Criminológico." Valencia: Tirant Lo Blanch.

García España, E. and Pérez Jiménez, F. 2004. "Reciente Evolución De La Delincuencia En Andalucía." Revista Electrónica De Ciencia Penal y Criminología:4.

García España, E., Pérez Jiménez, F., Benítez Jiménez, M.J., Instituto Andaluz Interuniversitario de Criminología, Observatorio de la Delincuencia en Andalucía and Fundación El Monte. 2006. La Delincuencia Según Las Víctimas :Un Enfoque Integrado a Partir De Una Encuesta De Victimización : Informe ODA 2006. Málaga: Instituto Andaluz Interuniversitario de Criminología.

Hale, C. 1996. "Fear of Crime: A Review of the Literature." International Review of Victimology 4:79-150.

Hearnden, I. and C. Magill. 2004. Decision-Making by House Burglars: Offenders' Perspectives. Home Office. Research, Development and Statistics Directorate.

Kershaw, C., S. Nicholas and A. Walker. 2008. "Crime in England and Wales 2007/08." Home Office Statistical Bulletin 7(08).

Landrove-Diaz, G. 1990. "Victimologia, Ed." Tirant Lo Blanch Derecho.

Maguire, M. and T. Bennett. 1982. Burglary in a Dwelling: The Offence, the Offender and the Victim. Heinemann London.

Mawby, RI. 2001. Burglary. Willan Publishing.

Medina, J. 2003. "Inseguridad Ciudadana, Miedo Al Delito y Policía En España." Revista Electrónica De Ciencia Penal y Criminología:05-03.

Middendorf, W. 1973. "The Case of August Sangret A Contribution to Historical Criminology." 12(1-2):13. 
Reppetto, T. A. 1974. Residential Crime. Ballinger Cambridge, MA.

Ruiz, L. C. Á. 2006. Delitos Contra El Patrimonio Aspectos Penales y Criminológicos" Especial Referencia a Badajoz": Aspectos Penales y Criminológicos. Editorial Visionnet.

Scarr, H. A. 1973. "Criminal Justice Research: Patterns of Burglary." US Department of Justice, Law Enforcement Assistance Administration, National Institute of Law Enforcement and Criminal Justice, Washington, DC.

Van Dijk, JJM and C. H. D. Steinmetz. 1983. "Victimisation Surveys: Beyond Measuring the Volume of Crime." Victimology 8(1-2):19.

Walsh, D. 1980. Break-Ins: Burglary from Private Houses. Constable London.

Webster, R. 2004. "Waltham Forest Crime and Disorder and Drug Audit 2004.".

\section{El autor:}

Francesc Reales Arnó es Subinspector del cuerpo de la policía de la Generalitat de Cataluña, Mossos d'Esquadra y su campo de interés es la prevención de la delincuencia.

Es licenciado en Filología Inglesa y licenciado en Criminología, posee un Master en Criminología en la especialidad de investigación criminal y es Diplomado en Estudios Avanzados en el doctorado de Sistema de Justicia Penal por la Universidad de Lleida. Actualmente está realizando la tesis doctoral en el área de la prevención situacional del delito, en concreto en el estudio de la revictimización en el delito de robo en vivienda. e-mail: freales@gmail.com 